\title{
Editorial
}

\section{Trends in hypertension}

\author{
Dayasagar Rao* \\ Senior Consultant Cardiologist, Krishna Institute of Medical Sciences, Hyderadad, AP, India.
}

Non-communicable diseases, especially cardiovascular diseases are major cause of death and disability in the world, even in the low and middle income countries. The International Case Control Studies-INTER HEART ${ }^{1}$ and INTER STROKE ${ }^{2}$ involving 52 countries, including those of low income reported that standard 10 risk factors account for $>90 \%$ of vascular events. But the population attributable risks are different in INTER HEART and INTER STROKE, with greater importance of lipids (49.2\%), diabetes $(9.9 \%)$ in INTER HEART and of hypertension (HTN) (34.6\%) in INTER STROKE.

The level of blood pressure (BP) at which to intervene is still debated by HTN experts. The suggested levels at which to intervene has changed from $120 / 80$ in 1942 , to $180 / 100$ in 1948 , and 150/90 in 1952. In 1959, World Health Organization (WHO) published a report about technique of measurement of BP and cut-off value of 160/95 which became a standard dividing line between normotension and HTN. Norman Kaplan stated that the dividing line be established on some rational basis and defined HTN as that level of BP at which benefits of action exceed the risks of inaction. This level can only be determined by longitudinal studies of cohort of patients over a long period of time (for identifying level at which cardiovascular risk occurs) and by intervention trials demonstrating benefits from BP reduction. Stamler et al. ${ }^{3}$ analysed all the US studies of correlation of level of BP with cardiovascular events and showed that both systolic BP (SBP) and diastolic BP (DBP) have continuous, graded, independent relationship to the clinical outcome variables of cardiovascular mortality and total mortality. The longitudinal study of Framingham reported continuum of risk of stroke and coronary artery disease with DBP, with no cut-off value at which risk was not present. Further intervention trials like Treatment Of Mild Hypertension Study (TOMHS) (BP $=140-159 / 90-95 \mathrm{mmHg}$ ) have shown better vascular outcome compared to placebo. Similar data was reported in Multiple Risk Factor Intervention Trial (MRFIT) with excess mortality above SBP of $120 \mathrm{mmHg}$.

\footnotetext{
*Corresponding author.

E-mail address: e-dsrvala@rediffmail.com
}

No prospective longitudinal study of correlating level of BP with clinical events exists in Asian countries. Most of the studies have been one time cross-sectional studies and in a few at different points of time showing trend of HTN prevalence over decades of time like in a paper published in this journal from Nepal. Striking feature of various prevalence studies has been progressive rise in SBP throughout life, with a difference of $20-30 \mathrm{mmHg}$ between early and late adulthood with less striking increase in DBP. In later years, the DBP may level off or slightly decline. The mean level of SBP and DBP are higher in men than women in early adulthood, but the difference narrows progressively and may even be reversed by the sixth or seventh decade.

Studies evaluating the prevalence of HTN in the same geographical area over decades have not been many in India. In an attempt to evaluate the changing trends of prevalence of HTN in India, Gupta et al. ${ }^{4}$ analysed epidemiological studies done in India from 1950 onwards till 1998. Most of these studies used WHO criteria $(>160 / 95 \mathrm{mmHg}$ ) for diagnosis of HTN, which showed increasing trend from $4.35 \%$ in Agra (Mathur 1963), 6.4\% in Rohtak (Gupta 1978) and 15.52\% in Bombay (Dalal 1980). Using Joint National Council (JNC) V criteria $(>140 / 90 \mathrm{mmHg}$ ) also showed steep increase in prevalence of HTN from 6.2\% (Delhi 1959), 30.9\% (Jaipur 1995), 44\% (Mumbai 1999), and 36\% (Chennai 2001). Bhopal et al. ${ }^{5}$ compared HTN prevalence rates in Indians, Pakistanis, and Bangladeshis in Britain and reported that HTN was more in Indians compared to other South Asian groups. In SHARE ${ }^{6}$ study, the prevalence of self-reported HTN amongst South Asians (12.5\%) was similar to Europeans (11\%) but lower than Chinese (15.9\%). Ahlawat et al. ${ }^{7}$ reported trends in HTN in Chandigarh-Urban North Indian-population over a 30-year period. The prevalence in 1968 was $19.99 \%$ in men and $24.8 \%$ in women which increased to $43.7 \%$ in men and $45.8 \%$ in women in 1997. Similar study was done in Jaipur, once in 1995 (Jaipur Heart Watch $1-\mathrm{JHW} 1^{8}$ ) and subsequently in 2002 $\left(\mathrm{JHW} 2^{9}\right.$ ). In JHW1, HTN was present in $29.5 \%$ men and $33.5 \%$ women as compared to $36.4 \%$ men and $37.5 \%$ women in JHW2, but age-adjusted prevalence rates are not significantly 
different in the two studies. Further, the age-adjusted prevalence of prehypertension (high normal BP 120-139/80-89) was not significantly different between the two studies. In this journal, cross-sectional study of prevalence of HTN in Nepal over a period of three decades showed tripling of prevalence even by WHO definition $(>160 / 95 \mathrm{mmHg}) .^{10}$ Further, there was good correlation of increased prevalence of HTN with obesity (body mass index) and salt consumption which emphasises the importance of lifestyle changes in prevalence of HTN.

This study from Nepal is done in rural Kathmandu (Bhadrabas and Alapot) in a small number of subjects using only single casual measurement BP using mercury manometer. Further, the study used different criteria for HTN at different points of time (WHO definition of 160/95 in 1980 and JNC criteria of 140/90 in 2006) and thus making the interpretation difficult. But comparison of mean SBP reveals an increase from $118.4 \mathrm{mmHg}$ in 1980 to $120.82 \mathrm{mmHg}$ in 2006 and an increase in mean DBP of $74.6 \mathrm{mmHg}$ to $80.14 \mathrm{mmHg}$. Although, these differences of $1-2 \mathrm{mmHg}$ may appear small, they have good correlation with changes in prevalence of HTN. An increase in mean BP is associated with increase in prevalence. In various cohorts of seven ${ }^{11}$ countries study, the trends in prevalence of HTN changed with changes in mean BP. In Dutch cohort, during the period 1974-1980, the average SBP increased by $2 \mathrm{mmHg}$ and DBP by $4 \mathrm{mmHg}$ and prevalence of HTN increased from $12.7 \%$ in 1974 to $17.8 \%$ in 1980 . Similar trends in prevalence of HTN were seen in Yugoslavian cohort. The Framingham ${ }^{12}$ study reported a decline in mean SBP and DBP over a period of 40 years that was associated with declining HTN prevalence. This study from Nepal also showed an increase in mean SBP and DBP with increased prevalence of HTN over a period of three decades.

Prevalence studies in USA between late 1970 and early 1990 , declined from $32 \%$ to $25 \%$, but the most recent survey data shows steady increase between 1988-1994 (23.9\% men and $26 \%$ in women) and $1999-2000$ (27.1\% men and $30.1 \%$ women) with prevalence of $28.6 \%$ in 2005 . There are ethnic differences observed in USA with almost $50 \%$ higher prevalence in African American adults compared with their white counterparts. International data indicate even higher prevalence of HTN in adults in some developed countries. Whereas the prevalence of HTN in Canada in adults (35-74 years) is $28 \%$, similar to USA in 1990, concurrent data from six European countries revealed an overall prevalence rate of $44 \%$ with lowest prevalence in Italy 38\% and highest in Germany 55\%. Meta analysis ${ }^{13}$ of various studies of prevalence of HTN across the globe revealed that an overall $26.4 \%$ of adult population in 2000 had HTN (26.6\% men and $26.1 \%$ women) and the number projected by 2025 will be $29.2 \%$. The estimated total number of adults with HTN in 2000 was 972 million-333 million in developed and 639 million in developing countries and the predicted number by 2025 is 1.56 billion. This study reveals important public health challenge and the potential for preventive and therapeutic strategies.

Essential HTN is considered to be due to interaction of genetic susceptibility and adverse environmental factors resulting in obesity (specially truncal), higher dietary salt intake, poor intake of potassium and fibre and physical inactivity. These environmental factors not only explain the ethnic differences and increase in prevalence of BP but are also targets for prevention of HTN, an important risk factor for vascular disease.

\section{References}

1. The INTERHEART study: case control study. Lancet 2004;364: 937-52.

2. The INTERSTROKE study: case control study. Lancet 2010;376: 112-23.

3. Stamler J, Stamler R, Neaton JD. Blood pressure, systolic and diastolic, and cardiovascular risks: US population data. Archives Internal Medicine 1993;153:598-615.

4. Gupta R, Al-odat NA, Gupta VP. Hypertension epidemiology in India: meta-analysis of 50-year prevalence rates and blood pressure trends. J Hum Hypertens 1996;10:465-72.

5. Bhopal R, Unwin R, White M, et al. Heterogeneity of coronary heart disease risk factors in Indian, Pakistani, Bangladeshi, and European origin populations: cross sectional study. BMJ 1999; 319:215-20.

6. Anand SS, Yusuf S, Vuksan V, et al. Differences in risk factors, atherosclerosis and cardiovascular disease between ethnic groups in Canada: The Study of Health Assessment and Risk in Ethnic Groups (SHARE). Lancet 2000;356:279-84.

7. Ahlawat SK, Singh MMC, Kumar R, Kumari S, Sharma BK. Time trends in the prevalence of hypertension and associated risk factors in Chandigarh. J Indian Med Assoc 2002;100:547-55.

8. Gupta R, Guptha S, Gupta VP, Prakash H. Prevalence and determinants of hypertension in the urban population of Jaipur in western India. J Hypertens 1995;13:1193-200.

9. Gupta R, Gupta VP, Sarna M, et al. Prevalence of coronary heart disease and risk factors in an urban Indian population. Indian Heart J 2002;54:59-66.

10. Vaidya A, Pathak RP, Pandey MR. Prevalence of hypertension in Nepalese community triples in 25 years: a repeat cross-sectional study in rural Kathmandu. Indian Heart J 2012;64:128-31.

11. Toshima H, Koga Y, Blackburn. Lessons for science from seven countries study: a 35-year collaborative experience in cardiovascular disease epidemiology. Springer-Verlag: Tokyo 1994.

12. Mostero A, D’Agostino RB, Silbershatz H, et al. Trends in prevalence of hypertension, antihypertensive therapy, and left ventricular hypertrophy from 1950 to 1989. N Engl J Med 1999;340: 1221-7.

13. Kearney PM, Whelton M, Reynolds K, et al. Global burden of hypertension: analysis of worldwide data. Meta analysis. Lancet 2005;365:217-23. 